\title{
Over- and under-prophylaxis for chemotherapy-induced (febrile) neutropenia relative to evidence-based guidelines is associated with differences in outcomes: findings from the MONITOR-GCSF study
}

\author{
Carsten Bokemeyer ${ }^{1}$ Pere Gascón ${ }^{2} \cdot$ Matti Aapro $^{3} \cdot$ Heinz Ludwig ${ }^{4}$. \\ Mario Boccadoro $^{5} \cdot$ Kris Denhaerynck $^{6,7}$ Michael Gorray $^{8}$ - Andriy Krendyukov ${ }^{8}$. \\ Ivo Abraham ${ }^{6,9}$ (D) Karen MacDonald $^{6}$
}

Received: 20 September 2016 / Accepted: 9 January 2017 /Published online: 22 January 2017

(C) The Author(s) 2017. This article is published with open access at Springerlink.com

\begin{abstract}
Purpose In the MONITOR-GCSF study of chemotherapyinduced (febrile) neutropenia with biosimilar filgrastim, $56.6 \%$ of patients were prophylacted according to amended EORTC guidelines, but $17.4 \%$ were prophylacted below and $26.0 \%$ above guideline recommendations.

Methods MONITOR-GCSF is a prospective, observational study of 1447 evaluable patients from 140 cancers centers in 12 European countries treated with myelosuppressive chemotherapy for up to 6 cycles receiving biosimilar GCSF prophylaxis. Patients were classified as under-, correctly-, or overprophylacted with GCSF relative to guideline recommendations
\end{abstract}

Ivo Abraham

iabraham@matrix45.com

Universitaetsklinikum Hamburg Eppendorf, Hamburg, Germany

2 Division of Medical Oncology, Department of Hematology-Oncology, Hospital Clínic de Barcelona, University of Barcelona, Barcelona, Spain

3 Institut Multidisciplinaire d'Oncologie, Clinique de Genolier, Genolier, Switzerland

4 Medizinische Abteilung I - Onkologie und Haematologie, Wilhelminenspital, Wien, Austria

5 Dipartimento di Oncologia e Ematologia, Azienda Ospedaliero Universitaria S. Giovanni Battista di Torino, Torino, Italy

6 Matrix45, 6159 W Sunset Rd, Tucson, AZ 85743, USA

7 Universitaet Basel, Basel, Switzerland

8 Hexal AG, Holzkirchen, Germany

9 Center for Health Outcomes and PharmacoEconomic Research, University of Arizona, Tucson, AZ, USA based on their chemotherapy risk, individual risk factors, and type of GCSF prophylaxis (primary versus secondary).

Results Differences between under- (17.4\%), correctly$(56.6 \%)$, or over-prophylacted $(26.0 \%)$ groups were found in terms of patient risk factors (age, performance status, history of $\mathrm{FN}$, comorbid conditions) as well as prophylaxis patterns (type of prophylaxis, day of GCSF initiation, and GCSF duration). Rates of chemotherapy-induced neutropenia (CIN) (all grades), FN, and CIN-related hospitalizations were consistently lower in over-prophylacted patients relative to under- and correctlyprophylacted patients. No differences were observed between under- and correctly-prophylacted patients except for CIN/FNrelated chemotherapy disturbances. No GCSF safety differences were found between groups (except for headaches).

Conclusions The real-world evidence provided by the MONITOR-GCSF study indicates that providing GCSF support may yield better CIN, FN, and CIN/FN-related hospitalization outcomes if patients are prophylacted at levels above guideline recommendations. Patients who are underprophylacted are at higher risk for disturbances to their chemotherapy regimens. Our findings support the guideline recommendation that $\mathrm{CIN} / \mathrm{FN}$ risk be assessed at the beginning of each chemotherapy cycle.

Keywords Chemotherapy-induced neutropenia $\cdot$ Febrile neutropenia - Granulocyte colony stimulating factor . Filgrastim $\cdot$ EP2006 $\cdot$ Biosimilar $\cdot$ Prophylaxis

\section{Introduction}

Evidence-based guidelines for the prophylaxis of chemotherapyinduced (CIN) and febrile neutropenia (FN) of the European 
Organization for Research and Treatment of Cancer (EORTC) [1] and the National Comprehensive Cancer Network (NCCN) [2] recommend that clinical decision-making be based on the relative myelotoxicity of patients' chemotherapy therapy regimens and the presence of potential risk factors. Prophylaxis with granulocyte colony-stimulating factors (GCSF) is indicated for patients treated with chemotherapy with an FN risk $\geq 20 \%$ and for patients receiving chemotherapy with an FN risk of 10-20\% if they also present with risk factors. No prophylaxis is recommended for patients given chemotherapy with an FN risk $<10 \%$.

The MONITOR-GCSF study was a pan-European multicenter longitudinal prospective study of practice patterns and outcomes associated with CIN/FN prophylaxis with biosimilar filgrastim (EP2006, Zarzio ${ }^{\circledR} /$ Zarxio $^{\circledR}$, Hexal AG/ Sandoz International $\mathrm{GmbH}$ ) that includes 1447 evaluable patients from 140 cancers centers in 12 European countries treated with myelosuppressive chemotherapy across a total of 6213 cycles [3, 4]. This study used the EORTC guidelines [1] as a framework, specifically the algorithm of evaluating the myelotoxicity of the chemotherapy regimen and the associated FN risk $(<10 \%, 10-20 \%, \geq 20 \%)$ as well as the presence of conditions with high risk (age $\geq 65$ years) and increased FN risk (advanced disease, history of FN, no antibiotic prophylaxis), as well as other factors associated with FN (poor performance and/or nutritional status, female gender, hemoglobin $<12 \mathrm{~g} / \mathrm{dL}$, renal, cardiovascular, or liver disease). This algorithm was amended by expert consensus to recommend secondary prophylaxis in patients who experienced a CIN or FN episode in a prior cycle and receiving low-risk $(<10 \%)$ or medium-risk (10-20\%) chemotherapy (Fig. 1). The amended algorithm also specified that secondary prophylaxis was not indicated for patients receiving chemotherapy regimens with $\geq 20 \%$ myelotoxicity or with $10-20 \%$ myelotoxicity but in the presence of patient risk factors (as primary prophylaxis should have been administered) or for patients treated with regimens with $<10 \%$ or $10-20 \%$ myelotoxicity but no CIN/FN in a prior cycle.

We used this amended algorithm to classify patients according to prophylaxis intensity level. As reported earlier, of 1444 classifiable patients, 817 (56.6\%) were correctlyprophylacted, $251(17.4 \%)$ were under-prophylacted, and 376 (26.0\%) were over-prophylacted (Fig. 1) [5]. Modeling analyses revealed that under-prophylaxis was an independent predictor of patients experiencing an $\mathrm{FN}$ episode or a CIN/FNrelated hospitalization [6]. In contrast, over-prophylaxis was associated with a lowered risk for a $\mathrm{CIN}$ grade 4 or $\mathrm{FN}$ episode or a CIN/FN-related hospitalization.

To further explore the impact of prophylaxis intensity below or above guideline-recommended levels, we performed analyses stratified by prophylaxis intensity (under/correctly/ over-prophylacted) that compare patients' in terms of demographics and clinical status at the start of chemotherapy, Zarzio ${ }^{\circledR}$ prophylaxis patterns, and clinical and safety outcomes. In keeping with our prior reports [5, 6], we distinguish between results using patients and results using cycles as the unit of analysis. The patient-level evaluations focus on outcomes "ever" experienced anytime during the whole period of chemotherapy and inform about patient outcomes across this line of chemotherapy. The cycle-level analyses target outcomes recorded during a particular cycle and from 1 cycle to the next, and inform about outcomes as patients progress through the cycles of chemotherapy.

\section{Methods}

The background and methodology of MONITOR-GCSF [3, 4] as well as the study sample's demographics and clinical status at baseline, Zarzio ${ }^{\circledR}$ prophylaxis, and outcomes [5] have been described elsewhere. We summarize below elements relevant to the present analyses.

\section{Design}

MONITOR-GCSF is a prospective, real-world, observational study of cancer patients receiving myelosuppressive chemotherapy, whose treating physicians prescribed $\mathrm{CIN} / \mathrm{FN}$ prophylaxis with biosimilar filgrastim (EP2006, Zarzio®) per their best clinical judgment. Eligible were adults (age $\geq 18$ ) with stages 3 or 4 breast, ovarian, bladder, or lung cancer; metastatic prostate cancer; and stages 3 or 4 diffuse large Bcell lymphoma or multiple myeloma. Patients were followed up for a maximum of six chemotherapy cycles. Patients were classified as to prophylaxis intensity according the schematic in Fig. 1.

\section{Outcomes}

The following outcomes were recorded at both the patientand cycle-levels: occurrence of an episode of CIN of any grade (CIN1/4), specified further as CIN grades 3 or 4 (CIN3/4), CIN grade 4 (CIN4), or an FN episode; CIN/FNrelated hospitalization or chemotherapy disturbance (dose reduction, delay in administration of chemotherapy, cancelation of administration of chemotherapy); and a (worst-case) composite index of any of these outcomes occurring.

\section{Specialized statistical issues}

The Patient risk score (PRS) is the weighted sum (range 0-11) of each of the eight patient risk factors associated with CIN/ FN specified in the EORTC guidelines [1] and was developed by consensus by four of the authors (C.B., P.G., M.A., H.L). Weights of three were assigned to age $\geq 65$ and history of prior $F N ; 1.5$ to advanced disease and poor performance and/or nutritional status; and 0.5 to no antibiotic prophylaxis, female 
Fig. 1 Treatment decision relative to EORTC guidelines

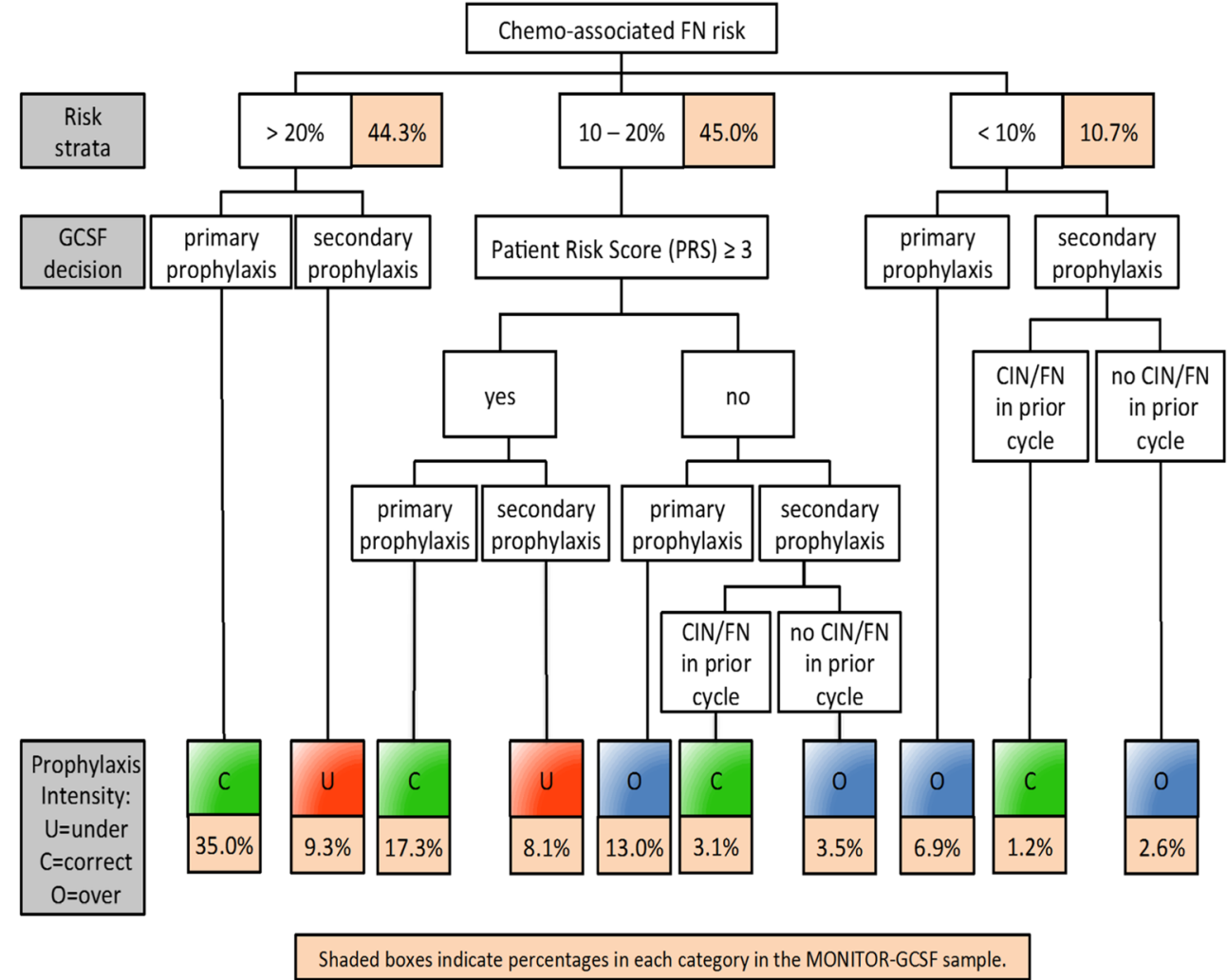

gender, hemoglobin $<12 \mathrm{~g} / \mathrm{dL}$, and renal, cardiovascular or liver disease. A PRS $\geq 3$ was used to consider a patient as being at elevated risk for CIN/FN (Fig. 1).

Cycle data were "nested" under patients and patients under centers, violating the assumption of statistical independence. Hence we applied generalized estimating equations (GEE) [7], which adjust standard errors based on within-cluster correlations, to estimate adjusted odds ratios (OR) and 95\% confidence intervals $(95 \% \mathrm{CI})$. We calculated ORs for each outcome for each prophylaxis intensity cohort separately to determine the odds of each outcome for each cohort, as well as in pairwise combinations, to contrast the relative odds of one prophylaxis intensity level against another level. Chemotherapy disturbances were estimated for the cycle after $($ lag $=1)$ the $\mathrm{CIN} / \mathrm{FN}$ event occurred.

\section{Results}

\section{Patients}

Of the 1444 patients who could be classified as to prophylaxis intensity, $817(56.6 \%)$ were correctlyprophylacted, $251(17.4 \%)$ were under-prophylacted, and $376(26.0 \%)$ were over-prophylacted (Fig. 1; Table 1). The three cohorts were similar in terms of gender and history of repeated infections (both $p=$ n.s.). They differed in terms of average age $(p<0.001)$, with underprophylacted patients older and over-prophylacted patients younger than correctly-prophylacted patients. The cohorts also varied in performance status $(p=0.011)$, with proportionately more over-prophylacted patients having ECOG 0/1 scores and more under-prophylacted patients having ECOG $1 / 2$ scores relative to correctlyprophylacted patients. When comparing the cohorts on the EORTC risk factors, no differences were observed in the proportions of female patients and those without antibiotic prophylaxis (both $\mathrm{p}=$ n.s.). However, there were proportionately more under-prophylacted patients age $\geq 65(p<0.001)$, with a history of FN $(p=0.029), \mathrm{Hb}$ $<12 \mathrm{~g} / \mathrm{dL}(p<0.001)$, and renal, cardiovascular, or liver disease $(p<0.001)$. Both under- and over-prophylacted patients had proportionately more patients with advanced disease ( $p=0.009$ ) but fewer with poor performance and/ or nutritional status $(p=0.005)$ compared to correctlyprophylacted patients. Mean PRS was highest among under-prophylacted and lowest among over-prophylacted patients $(p<0.001)$.

The over-prophylacted cohort included significantly more patients with solid and fewer with hematological malignancies compared to the under- and correctly-prophylacted cohorts ( $p=0.009)$, who did not differ from each other $(p=$ n.s.). Both the under- and over-prophylacted cohorts had proportionately more patients previously treated with chemotherapy 
Table 1 Patient demographics and clinical status, cancer and $\mathrm{CIN} / \mathrm{FN}$ history, and management

\begin{tabular}{|c|c|c|c|c|}
\hline & $\begin{array}{l}\text { Under } \\
251(17.4 \%)\end{array}$ & $\begin{array}{l}\text { Correct } \\
817(56.6 \%)\end{array}$ & $\begin{array}{l}\text { Over } \\
376(26.0 \%)\end{array}$ & $p$ \\
\hline \multicolumn{5}{|l|}{ Demographics and clinical status } \\
\hline Gender & & & & n.s. \\
\hline Male & $45.0 \%$ & $37.2 \%$ & $37.8 \%$ & \\
\hline Female & $55.0 \%$ & $62.8 \%$ & $62.2 \%$ & \\
\hline Age $(\mathrm{M} \pm \mathrm{SD}, \mathrm{Mdn})$ & $\begin{array}{l}65.2 \pm 11.0 \\
67\end{array}$ & $\begin{array}{c}61.8 \pm 12.5 \\
63\end{array}$ & $57.7 \pm 9.5,58$ & $<0.001$ \\
\hline \multicolumn{5}{|l|}{ ECOG performance status } \\
\hline 0 & $31.5 \%$ & $40.7 \%$ & $47.4 \%$ & \\
\hline 1 & $57.8 \%$ & $46.1 \%$ & $46.5 \%$ & \\
\hline 2 & $9.9 \%$ & $10.9 \%$ & $4.2 \%$ & 0.011 \\
\hline 3 & $0.9 \%$ & $2.4 \%$ & $1.7 \%$ & \\
\hline 4 & $0.0 \%$ & $0.0 \%$ & $0.3 \%$ & \\
\hline History of repeated infections & $4.3 \%$ & $2.3 \%$ & $2.0 \%$ & n.s. \\
\hline \multicolumn{5}{|l|}{ FN risk factors (EORTC) } \\
\hline \multicolumn{5}{|l|}{ High risk } \\
\hline Age $\geq 65$ years & $61.4 \%$ & $46.1 \%$ & $17.3 \%$ & $<0.001$ \\
\hline \multicolumn{5}{|l|}{ Increased risk } \\
\hline Advanced disease $^{\mathrm{a}}$ & $15.3 \%$ & $11.2 \%$ & $18.2 \%$ & 0.009 \\
\hline History of FN & $6.5 \%$ & $0.8 \%$ & $1.8 \%$ & 0.029 \\
\hline No antibiotic prophylaxis & $90.0 \%$ & $85.4 \%$ & $91.6 \%$ & n.s. \\
\hline \multicolumn{5}{|l|}{ Other factors } \\
\hline $\begin{array}{l}\text { Poor performance and/or nutritional } \\
\text { status }\end{array}$ & $11.8 \%$ & $16.2 \%$ & $8.5 \%$ & 0.005 \\
\hline Female gender & $55.0 \%$ & $62.8 \%$ & $62.2 \%$ & n.s. \\
\hline Hemoglobin $<12 \mathrm{~g} / \mathrm{dL}$ & $60.0 \%$ & $34.4 \%$ & $39.4 \%$ & $<0.001$ \\
\hline Renal, cardiovascular, or liver disease & $31.6 \%$ & $24.7 \%$ & $13.5 \%$ & $<0.001$ \\
\hline Patient risk score $(\mathrm{M} \pm \mathrm{SD}, \mathrm{Mdn})$ & $3.8 \pm 2.0,4$ & $2.9 \pm 2.0,3$ & $2.0 \pm 1.6,1.5$ & $<0.001$ \\
\hline \multicolumn{5}{|l|}{ Cancer } \\
\hline Tumor type & & & & 0.009 \\
\hline Solid & $72.5 \%$ & $73.7 \%$ & $88.0 \%$ & \\
\hline Hematological & $27.5 \%$ & $26.3 \%$ & $12.0 \%$ & \\
\hline \multicolumn{5}{|l|}{ Prior treatments } \\
\hline Chemotherapy & $37.1 \%$ & $26.9 \%$ & $39.1 \%$ & 0.004 \\
\hline Of these adjuvant in metastatic setting & $41.8 \%$ & $48.2 \%$ & $50.4 \%$ & n.s. \\
\hline Of these prior lines of chemo & $48.1 \%$ & $47.7 \%$ & $56.1 \%$ & n.s. \\
\hline 1 & $42.1 \%$ & $48.9 \%$ & $60.3 \%$ & \\
\hline 2 & $31.6 \%$ & $27.3 \%$ & $24.7 \%$ & n.s. \\
\hline$\geq 3$ & $26.3 \%$ & $23.9 \%$ & $15.1 \%$ & \\
\hline Radiation therapy & $19.5 \%$ & $16.9 \%$ & $23.1 \%$ & n.s. \\
\hline Chemotoxicity & & & & $<0.001$ \\
\hline$<10 \%$ & $0.0 \%$ & $2.1 \%$ & $36.4 \%$ & \\
\hline $10-20 \%$ & $46.6 \%$ & $36.0 \%$ & $63.6 \%$ & \\
\hline$\geq 20 \%$ & $53.4 \%$ & $61.9 \%$ & $0.0 \%$ & \\
\hline
\end{tabular}

${ }^{\text {a }}$ Stage 4 (stage 3 if multiple myeloma) and prior chemotherapy in metastatic setting n.s. not significant
( $p=0.004)$; however, there were no differences between cohorts as to whether this was in the adjuvant or meta-static setting, nor the number of prior lines of chemotherapy (all $p=$ n.s.). Cohorts had similar rates of prior radiation therapy
( $p=$ n.s.). There was an association between prophylaxis intensity and chemotoxicity. All over-prophylacted patients were treated with regimens with $<10 \%(36.4 \%)$ and 10-20\% FN risk $(63.6 \%)$, whereas all under-prophylacted patients 
received chemotherapy with $10-20 \%(46.6 \%)$ and $\geq 20 \%$ (53.4\%) FN risk. $61.9 \%$ of correctly-prophylacted patients were administered regimens with $\geq 20 \%$ FN risk $(p<0.001)$.

\section{Prophylaxis patterns}

All under-prophylacted patients received secondary prophylaxis only, almost all $(92.4 \%)$ of correctly-prophylacted patients had primary prophylaxis, whereas $76.6 \%$ of overprophylacted patients were given primary and $23.4 \%$ secondary prophylaxis $(p<0.001)$ (Table 2$)$. The median day of Zarzio ${ }^{\circledR}$ initiation was the second day after chemotherapy; however, the mean initiation day for over-prophylacted patients was 2.70 days post-chemotherapy, compared to 2.99 for under- and 3.26 for correctly-prophylacted patients ( $p=0.001)$. Further, $19.5 \%$ of over-prophylacted patients were initiated on the day of chemotherapy completion, compared to 12.1 and $11.2 \%$ of, respectively, under- and correctlyprophylacted patients. Despite a similar median prophylaxis duration of 5 days across all three cohorts, mean duration was shortest for over-prophylacted and longest for underprophylacted patients $(p<0.001)$. Cohorts did not differ in terms of proportions of patients given $30 \mathrm{MIU} /$ day versus $40 \mathrm{MIU} /$ day of Zarzio®.

\section{Outcomes}

Consistently, whether in the patient- or the cycle-level analyses, the three cohorts differed overall in the observed rates of CIN1/4, CIN3/4, CIN4, and FN, CIN/FN-related hospitalizations, and the composite outcome score (all $p \leq 0.001$ ) (Table 3). The proportions of patients experiencing CIN/FNrelated chemotherapy disturbances were not statistically different across cohorts $(p=$ n.s.); however, the proportion of cycles with chemotherapy disturbances was highest among under-prophylacted patients $(p=0.032)$.

Pairwise contrast analyses at the patient-level showed that, generally, the likelihood of CIN and FN episodes anytime during chemotherapy did not differ between under- and correctly-prophylacted patients (all $p=$ n.s.) (Table 4). However, over-prophylacted patients were less likely to experience $\mathrm{CIN} / \mathrm{FN}$ than correctly-prophylacted patients ever during chemotherapy (all $p<0.001$ ) or in any given cycle ( $p<0.001$ for all CIN, $p=0.004$ for FN). Compared to over-prophylacted patients, under-prophylacted patients had a greater likelihood of $\mathrm{CIN}$ and FN anytime during chemotherapy ( $p=0.044$ to $p<0.001$ ).

Pairwise contrast analyses at the cycle-level revealed that the likelihood of CIN/FN in a given cycle did not differ between under- and correctly-prophylacted patients (all $p=$ n.s.) (Table 4). Over-prophylacted patients were less likely to experience $\mathrm{CIN} / \mathrm{FN}$ than correctly-prophylacted patients in any given cycle $(p<0.001$ for all CIN, $p=0.004$ for FN). Under- prophylacted patients had a greater likelihood of CIN and FN in any given cycle compared to over-prophylacted patients $(p=0.025$ to $p<0.001)$.

\section{Safety}

With the exception of proportionately fewer underprophylacted patients experiencing headaches $(p=0.027)$, differences between cohorts in the rates of patients reporting clinical events of interest during the course of chemotherapy were not statistically significant (all $p=$ n.s.) (Table 5). Reported rates of adverse drug reactions over 6142 cycles were statistically similar across the three cohorts $(p=$ n.s.).

\section{Discussion}

By the time of the approval of Zarzio ${ }^{\circledR}$ by the European Medicines Agency in 2008 and the launch of the MONITOR-GCSF study in 2010, two decades of evidence with reference filgrastim had accumulated. Much of this was summarized in, for example, the original [8] and updated EORTC guidelines [1] and in systematic reviews and meta-analyses [9-16]. In the process, it became apparent that the normative, trial-based prophylaxis pattern of treating with standard GCSFs through the nadir of the absolute neutrophil count (ANC) was being replaced with shorter regimens varying across tumor types in the average number of injections [17].

The relative maturity of the clinical experience with standard GCSFs in routine clinical practice was documented in our earlier report on treatment patterns and outcomes in the MONITOR-GCSF study [5]. The median duration of prophylaxis was 5 days, and there were no differences in mean duration between patients receiving primary and those receiving secondary prophylaxis and when comparing oncological versus hematological patients. However, mean durations were progressively longer as the relative FN risk of patients' chemotherapy regimens rose from $<10 \%(M=4.59$ days $)$ to 10 $20 \%(M=4.98)$ and $\geq 20 \%(M=5.33)(p<0.001)$. Also noted were differences in prophylaxis intensity relative to the amended EORTC guidelines used in the MONITOR-GCSF study. Slightly over half of patients $(56.6 \%)$ were prophylacted relative to the guidelines, about one in six patients received less prophylaxis than recommended, and about a quarter were administered more prophylaxis than advised by the guidelines. These deviations from evidence-based guidelines in daily clinical practice suggest either questionable clinical practice or may provide new real-world data to integrate into guidelines as the evidence migrates from initial RCTbased findings to incorporating real-world data from a mature clinical experience base in GCSF support and CIN/FN prophylaxis. 
Table 2 Zarzio® prophylaxis patterns

\begin{tabular}{|c|c|c|c|c|}
\hline Type of prophylaxis & Under & Correct & Over & $p$ \\
\hline Primary & $0.0 \%$ & $92.4 \%$ & $76.6 \%$ & $<0.001$ \\
\hline Secondary & $100.0 \%$ & $7.6 \%$ & $23.4 \%$ & \\
\hline Dose & Under & Correct & Over & \\
\hline $30 \mathrm{MIU} /$ day & $51.4 \%$ & $53.4 \%$ & $55.1 \%$ & n.s. \\
\hline $48 \mathrm{MIU} /$ day & $48.6 \%$ & $46.6 \%$ & $44.9 \%$ & \\
\hline Day of initiation ${ }^{\mathrm{a}}$ & Under & Correct & Over & \\
\hline 0 (during chemo) & $12.1 \%$ & $11.2 \%$ & $19.5 \%$ & $<0.001$ \\
\hline 1-3 (according to guidelines) & $59.3 \%$ & $55.2 \%$ & $56.8 \%$ & \\
\hline \multirow[t]{2}{*}{4 or more (late) } & $28.6 \%$ & $33.5 \%$ & $23.7 \%$ & \\
\hline & Mean & SD & Median & \\
\hline By prophylaxis decision & & & & 0.001 \\
\hline Under & 2.99 & 3.01 & 2 & \\
\hline Correct & 3.26 & 2.99 & 2 & \\
\hline Over & 2.70 & 2.92 & 2 & \\
\hline \multicolumn{5}{|c|}{ Duration of prophylaxis at baseline (days) } \\
\hline & Under & Correct & Over & \\
\hline 1 & $3.3 \%$ & $2.6 \%$ & $5.3 \%$ & \\
\hline 2 & $5.3 \%$ & $3.4 \%$ & $10.5 \%$ & \\
\hline 3 & $17.9 \%$ & $12.1 \%$ & $15.5 \%$ & \\
\hline 4 & $3.7 \%$ & $7.7 \%$ & $4.7 \%$ & \\
\hline 5 & $41.5 \%$ & $46.8 \%$ & $42.8 \%$ & \\
\hline 6 & $5.3 \%$ & $7.4 \%$ & $6.4 \%$ & \\
\hline 7 & $11.0 \%$ & $11.5 \%$ & $9.1 \%$ & \\
\hline 8 & $3.3 \%$ & $1.9 \%$ & $2.2 \%$ & \\
\hline 9 & $0.8 \%$ & $1.5 \%$ & $0.6 \%$ & \\
\hline 10 & $2.0 \%$ & $2.2 \%$ & $0.8 \%$ & \\
\hline 11 & $1.2 \%$ & $0.4 \%$ & $0.3 \%$ & \\
\hline 12 & $1.2 \%$ & $0.4 \%$ & $0.0 \%$ & \\
\hline 13 & $0.0 \%$ & $0.3 \%$ & $0.3 \%$ & \\
\hline 14 & $2.9 \%$ & $1.6 \%$ & $1.4 \%$ & \\
\hline \multirow[t]{2}{*}{$\geq 15$} & $0.8 \%$ & $0.2 \%$ & $0.3 \%$ & \\
\hline & Mean & $\mathrm{SD}$ & Median & \\
\hline By prophylaxis decision & & & & $<0.001$ \\
\hline under & 5.44 & 2.70 & 5 & \\
\hline correct & 5.20 & 2.20 & 5 & \\
\hline over & 4.72 & 2.33 & 5 & \\
\hline Duration (all visits) & Under & Correct & Over & \\
\hline 1-3 days & $23.7 \%$ & $18.0 \%$ & $29.1 \%$ & 0.001 \\
\hline 4-5 days & $47.1 \%$ & $55.0 \%$ & $50.7 \%$ & \\
\hline $6+$ days & $29.2 \%$ & $27.0 \%$ & $20.2 \%$ & \\
\hline
\end{tabular}

${ }^{\text {a }}$ Zarzio® initiation expressed in days after chemotherapy

0 same day, 11 day after, 22 days after, etc. $n . s$. not significant
The analyses stratified by prophylaxis intensity reported here revealed that, compared to correctly-prophylacted patients, under-prophylacted patients were at no greater risk for experiencing CIN (all grades) and FN episodes, nor for CIN/ FN-related hospitalizations, over the course of their chemotherapy regimen; though they were at increased risk for disturbances to this regimen. Relative to over-prophylacted patients, under-prophylacted patients were at significantly higher risk for adverse outcomes over the period of chemotherapy, including a twofold increased risk for disruptions to their chemotherapy regimen and a threefold greater likelihood of CIN/FN-related hospitalizations. The apparent incremental protective effect of over-prophylaxis was also evident from comparisons to correctly-prophylacted patients. The odds of 
Table 3 Clinical outcomes at the patient and cycle levels by prophylaxis decision

\begin{tabular}{|c|c|c|c|c|c|c|c|}
\hline & \multicolumn{2}{|l|}{ Under } & \multicolumn{2}{|l|}{ Correct } & \multicolumn{2}{|l|}{ Over } & \multirow[t]{2}{*}{$p$} \\
\hline & $\%$ & $95 \% \mathrm{CI}$ & $\%$ & $95 \% \mathrm{CI}$ & $\%$ & $95 \% \mathrm{CI}$ & \\
\hline \multicolumn{8}{|l|}{ Unit of analysis, patient } \\
\hline \multicolumn{8}{|l|}{ Neutropenia episodes } \\
\hline CIN grades 1 through 4 & $41.0 \%$ & $(32.7 \%-49.9 \%)$ & $39.9 \%$ & $(32.8 \%-47.4 \%)$ & $19.7 \%$ & $(14.4 \%-26.3 \%)$ & $<0.001$ \\
\hline CIN grades 3 or 4 & $24.3 \%$ & $(18.3 \%-31.6 \%)$ & $27.4 \%$ & $(21.3 \%-34.5 \%)$ & $12.5 \%$ & $(8.5 \%-18.1 \%)$ & $<0.001$ \\
\hline CIN grade 4 & $12.0 \%$ & $(8.2 \%-17.2 \%)$ & $16.8 \%$ & $(12.4 \%-22.3 \%)$ & $6.4 \%$ & $(3.9 \%-10.3 \%)$ & $<0.001$ \\
\hline FN & $5.2 \%$ & $(2.6 \%-10.0 \%)$ & $8.0 \%$ & $(6.1 \%-10.3 \%)$ & $2.1 \%$ & $(1.1 \%-4.1 \%)$ & $<0.001$ \\
\hline CIN/FN-related hospitalizations & $8.0 \%$ & $(4.8 \%-12.9 \%)$ & $7.1 \%$ & $(5.0 \%-10.0 \%)$ & $2.7 \%$ & $(1.4 \%-4.9 \%)$ & 0.001 \\
\hline CIN/FN-related chemotherapy disturbances ${ }^{a}$ & $14.7 \%$ & $(10.4 \%-20.5 \%)$ & $8.8 \%$ & $(6.4 \%-12.1 \%)$ & $7.7 \%$ & $(4.9 \%-11.9 \%)$ & n.s. \\
\hline CIN/FN-related composite outcome $\mathrm{b}^{\mathrm{b}}$ & $24.7 \%$ & $(18.5 \%-32.2 \%)$ & $26.0 \%$ & $(21.2 \%-31.4 \%)$ & $13.0 \%$ & $(9.4 \%-17.8 \%)$ & $<0.001$ \\
\hline \multicolumn{8}{|l|}{ Unit of analysis, cycle } \\
\hline \multicolumn{8}{|l|}{ Neutropenia episodes } \\
\hline CIN grades 1 through 4 & $17.9 \%$ & $(14.7 \%-21.7 \%)$ & $16.0 \%$ & $(14.3 \%-17.9 \%)$ & $8.3 \%$ & $(6.4 \%-10.7 \%)$ & $<0.001$ \\
\hline CIN grades 3 or 4 & $9.5 \%$ & $(7.2 \%-12.4 \%)$ & $9.4 \%$ & $(8.2 \%-10.9 \%)$ & $3.8 \%$ & $(2.8 \%-5.3 \%)$ & $<0.001$ \\
\hline $\mathrm{CIN}$ grade 4 & $4.0 \%$ & $(2.8 \%-5.8 \%)$ & $4.8 \%$ & $(4.0 \%-5.8 \%)$ & $1.7 \%$ & $(1.1 \%-2.7 \%)$ & $<0.001$ \\
\hline FN & $1.6 \%$ & $(0.9 \%-2.9 \%)$ & $1.7 \%$ & $(1.3 \%-2.2 \%)$ & $0.5 \%$ & $(0.2 \%-1.1 \%)$ & $<0.001$ \\
\hline CIN/FN-related hospitalizations & $2.5 \%$ & $(1.6 \%-4.1 \%)$ & $1.6 \%$ & $(1.2 \%-2.2 \%)$ & $0.6 \%$ & $(0.3 \%-1.1 \%)$ & $<0.001$ \\
\hline CIN/FN-related chemotherapy disturbances ${ }^{a}$ & $4.2 \%$ & $(3.1 \%-5.7 \%)$ & $2.4 \%$ & $(1.9 \%-3.0 \%)$ & $2.3 \%$ & $(1.6 \%-3.4 \%)$ & 0.032 \\
\hline CIN/FN-related composite outcome $\mathrm{b}^{\mathrm{b}}$ & $8.6 \%$ & $(6.7 \%-11.0 \%)$ & $7.5 \%$ & $(6.5 \%-8.6 \%)$ & $3.9 \%$ & $(2.9 \%-5.3 \%)$ & $<0.001$ \\
\hline
\end{tabular}

${ }^{a}$ Type of chemotherapy disturbances are not mutually exclusive. Any patient may have experienced more than one type. Measured with 1 cycle lag

${ }^{\mathrm{b}}$ Includes any occurrence of CIN grade 4, FN, CIN/FN-related hospitalization, and/or CIN/FIN-related chemotherapy disturbance. CI confidence interval n.s. not significant

CIN or FN episodes during the course of chemotherapy were significantly lowered, as were the odds for hospitalization. This protective effect did not extend to preventing disruptions to the chemotherapy regimen. As a caution, note that these and similar results about prophylaxis intensity and CIN/FN outcomes were obtained from association-based analyses.

The relative robustness of these findings at the patient-level were confirmed in the cycle-level analyses. The likelihood of $\mathrm{CIN}$ and FN episodes and CIN/FN-related hospitalizations during a given cycle was not statistically different in underprophylacted patients when compared to correctlyprophylacted patients, but was statistically different relative to over-prophylacted patients. The likelihood of chemotherapy regimens being changed in a subsequent cycle was greater for under-prophylacted patients compared to other patients, but not for correctly-prophylacted patients. The relative robustness of both the patient- and cycle-level findings was underscored in the analyses of the composite outcome, which was an index of the "worst case scenario" of experiencing CIN grade $4, \mathrm{FN}, \mathrm{CIN} / \mathrm{FN}-$ related hospitalizations, and/or CIN/ FN-related chemotherapy disturbances ever during the course of chemotherapy or from 1 cycle to the next.

The patient- and cycle-level analyses of prophylaxis intensity draw attention to some well-established CIN/FN risk factors that are ignored in some cases leading to underprophylaxis or are emphasized in other cases leading to prophylaxis patterns "above" the guideline recommendation. These include age 65 years or older, ECOG performance score, tumor type, prior chemotherapy, and prior history of FN.

Note in this regard that the cycle-level analyses affirmed that CIN/FN outcomes may be a function of the cycle of chemotherapy, not just of the chemotherapy regimen in general. The results from these analyses enable clinicians to evaluate the risk of a CIN/FN episode at the start of each cycle, not just at the start of chemotherapy. This is consistent with the guideline recommendations to re-evaluate the risk of CIN/FN at the beginning of each cycle and to take the necessary precautions to prevent adverse $\mathrm{CIN} / \mathrm{FN}$ outcomes.

The findings that, generally, under- and correctlyprophylacted patients were at similar risk of adverse CIN/FN outcomes (except for chemotherapy disturbances) may seem counter-intuitive as one would expect that the former would have worse outcomes than the latter. Certainly, underprophylaxis is not indicated in general, in fact, the finding confirms the general need for prophylaxis. Clinicians' decision-making may play a role here, as they may choose to deviate from guideline recommendations, rely on clinical experience, determine CIN/FN prophylaxis on a case-by-case basis, and, as a result, under-prophylact some patients treated with less myelotoxic regimens, better clinical and performance status, and fewer if any risk factors. In fact, 
Table 4 Pairwise contrast odds ratios for clinical outcomes as a function of prophylaxis intensity at the patient and cycle levels

\begin{tabular}{|c|c|c|c|c|c|c|c|c|c|}
\hline & \multicolumn{3}{|c|}{$\begin{array}{l}\text { Under- versus correctly- } \\
\text { prophylacted }\end{array}$} & \multicolumn{3}{|c|}{$\begin{array}{l}\text { Over-versus correctly- } \\
\text { prophylacted }\end{array}$} & \multicolumn{3}{|c|}{$\begin{array}{l}\text { Under-versus over- } \\
\text { prophylacted }\end{array}$} \\
\hline & OR & $95 \% \mathrm{CI}$ & $p$ & OR & $95 \% \mathrm{CI}$ & $p$ & OR & $95 \% \mathrm{CI}$ & $p$ \\
\hline \multicolumn{10}{|l|}{ Unit of analysis, patient } \\
\hline \multicolumn{10}{|l|}{ Neutropenia episodes } \\
\hline CIN grades 1 through 4 & 1.048 & $0.720-1.527$ & n.s. & 0.369 & $0.261-0.522$ & $<0.001$ & 2.840 & $1.859-4.339$ & $<0.001$ \\
\hline $\mathrm{CIN}$ grades 3 or 4 & 0.850 & $0.571-1.266$ & n.s. & 0.378 & $0.261-0.547$ & $<0.001$ & 2.247 & $1.378-3.665$ & 0.001 \\
\hline CIN grade 4 & 0.674 & $0.420-1.081$ & n.s. & 0.338 & $0.206-0.555$ & $<0.001$ & 1.991 & $1.111-3.569$ & 0.021 \\
\hline FN & 0.632 & $0.301-1.326$ & n.s. & 0.252 & $0.121-0.524$ & $<0.001$ & 2.513 & $1.025-6.157$ & 0.044 \\
\hline CIN/FN-related hospitalizations & 1.133 & $0.640-2.007$ & n.s. & 0.358 & $0.195-0.657$ & $<0.001$ & 3.169 & $1.555-6.457$ & 0.002 \\
\hline CIN/FN-related chemotherapy disturbances ${ }^{\mathrm{a}}$ & 1.789 & $1.141-2.806$ & 0.011 & 0.865 & $0.553-1.353$ & n.s. & 2.069 & $1.150-3.723$ & 0.015 \\
\hline CIN/FN-related composite outcome $\mathrm{b}^{\mathrm{b}}$ & 0.936 & $0.627-1.398$ & n.s. & 0.428 & $0.293-0.624$ & $<0.001$ & 2.189 & $1.375-3.486$ & 0.001 \\
\hline \multicolumn{10}{|l|}{ Unit of analysis, cycle } \\
\hline \multicolumn{10}{|l|}{ Neutropenia episodes } \\
\hline CIN grades 1 through 4 & 1.147 & $0.875-1.503$ & n.s. & 0.475 & $0.348-0.648$ & $<0.001$ & 2.415 & $1.674-3.484$ & $<0.001$ \\
\hline CIN grades 3 or 4 & 1.006 & $0.718-1.411$ & n.s. & 0.381 & $0.262-0.555$ & $<0.001$ & 2.638 & $1.679-4.146$ & $<0.001$ \\
\hline CIN grade 4 & 0.834 & $0.541-1.285$ & n.s. & 0.348 & $0.213-0.569$ & $<0.001$ & 2.395 & $1.323-4.333$ & 0.004 \\
\hline FN & 0.926 & $0.482-1.779$ & n.s. & 0.301 & $0.132-0.686$ & 0.004 & 3.081 & $1.150-8.254$ & 0.025 \\
\hline CIN/FN-related hospitalizations & 1.588 & $0.895-2.818$ & n.s. & 0.354 & $0.174-0.718$ & 0.004 & 4.489 & $2.008-10.033$ & $<0.001$ \\
\hline CIN/FN-related chemotherapy disturbances ${ }^{\mathrm{a}}$ & 1.824 & $1.216-2.734$ & 0.004 & 0.991 & $0.634-1.550$ & n.s. & 1.840 & $1.124-3.012$ & 0.015 \\
\hline CIN/FN-related composite outcome $e^{b}$ & 1.169 & $0.855-1.599$ & n.s. & 0.504 & $0.357-0.713$ & $<0.001$ & 2.318 & $1.532-3.505$ & $<0.001$ \\
\hline
\end{tabular}

${ }^{\text {a }}$ Type of chemotherapy disturbances are not mutually exclusive. Any patient may have experienced more than one type. Measured with 1 cycle lag

${ }^{\mathrm{b}}$ Includes any occurrence of CIN grade 4, FN, CIN/FN related hospitalization, and/or CIN/FIN-related chemotherapy disturbancen.s. not significant

proportionately more under-prophylacted patients were male, in poorer health compared to correctly-prophylacted patients. over the age of 65, with ECOG scores of 1 or 2, and generally Despite these differences, the prophylaxis patterns of both

Table 5 Safety outcomes by prophylaxis decision

\begin{tabular}{lllll}
\hline & Under & Correct & Over & $p$ \\
\hline Clinical Events (patient level, $n=1447)$ & & & & \\
Bone pain & $23.6 \%$ & $28.5 \%$ & $25.0 \%$ & n.s. \\
Thrombocytopenia & $19.4 \%$ & $17.4 \%$ & $17.7 \%$ & n.s. \\
Serum LDH increase & $16.6 \%$ & $20.2 \%$ & $14.4 \%$ & n.s. \\
Muscle pain & $12.1 \%$ & $16.8 \%$ & $16.1 \%$ & n.s. \\
Joint pain & $11.6 \%$ & $15.9 \%$ & $15.6 \%$ & n.s. \\
Serum GGT increase & $12.8 \%$ & $14.9 \%$ & $15.4 \%$ & n.s. \\
Serum ALP increase & $11.9 \%$ & $13.3 \%$ & $15.3 \%$ & n.s. \\
Other neurological symptoms & $8.7 \%$ & $7.5 \%$ & $7.4 \%$ & n.s. \\
Headache & $3.4 \%$ & $8.9 \%$ & $7.3 \%$ & 0.027 \\
Blood uric acid increase & $9.3 \%$ & $7.7 \%$ & $5.0 \%$ & n.s. \\
Confusion/altered mental status & $3.5 \%$ & $2.6 \%$ & $4.6 \%$ & n.s. \\
Epistaxis & $1.8 \%$ & $2.2 \%$ & $2.5 \%$ & n.s. \\
Bleeding other than GI, skin hemorrhage & $2.6 \%$ & $2.0 \%$ & $2.8 \%$ & n.s. \\
Splenomegaly & $1.3 \%$ & $1.2 \%$ & $0.3 \%$ & n.s. \\
GI bleeding & $0.4 \%$ & $0.9 \%$ & $0.3 \%$ & n.s. \\
Skin hemorrhage & $0.4 \%$ & $0.8 \%$ & $0.3 \%$ & n.s. \\
Adverse drug reactions (cycle-level, $n=6142)$ & $2.3 \%$ & $1.8 \%$ & $1.5 \%$ & n.s. \\
\hline
\end{tabular}

$A L P$ alkaline phosphatase, GGT gamma glutamyl transpeptidase, GI gastro intestinal, $L D H$ lactate dehydrogenase n.s. not significant 
cohorts were similar in terms of Zarzio® dose, day of prophylaxis initiation, duration of prophylaxis, except that all underprophylacted patients were on secondary, and virtually all correctly-prophylacted patients were on primary prophylaxis.

Our findings point at the relative benefit of prophylacting at a higher intensity than recommended in the guidelines. This does not mean that over-prophylaxis is indicated across-the-board, but may merit consideration for selected patients. Proportionally, over-prophylacted patients tended to be younger; with no or minimal impairment in performance status; with only a few presenting with comorbid renal, cardiovascular, or liver; with lower Patient Risk Scores; with mainly solid tumors in more advanced stages of disease; having received a prior line of chemotherapy; and currently being treated with regimens with $<10 \%$ or $10-20 \%$ FN risk. For instance, onethird of patients $(n=466,32.2 \%)$ in the study had stages III or IV breast cancer. Of the 408 breast cancer patients who had an ECOG score of 0 or 1, 28.4\% were overprophylacted, slightly higher than (and contributing to) the full sample rate of $26.0 \%$. However, the overprophylaxis rate was $37.3 \%$ in stage IV breast cancer patients with ECOG score of 0 or 1 , and $36.3 \%$ in stage III patients with an ECOG score of 0 .

Over-prophylaxis may indicate a "playing-it-sure-andsafe" approach among clinicians, by focusing on subgroups of patients with a more balanced profile of risk factors in which a more intense prophylaxis approach is believed to lead to better outcomes. In contrast to the perhaps more benign (relatively speaking, that is) profile of over-prophylacted patients, under-prophylacted patients were generally the opposite: proportionately older, more impaired in performance, with more of them presenting with major comorbid disease, with more of them being treated for a hematological malignancy, with $\geq 2$ prior lines of chemotherapy, and currently receiving myelotoxic chemotherapy regimens with 10-20\% or $\geq 20 \% \mathrm{FN}$ risk. Of concern, this may reflect a trend in routine clinical practice to ignore the interaction of patient risk factors with the myelotoxicity of their chemotherapy regimens, leading to inadequate CIN/FN prophylaxis. Worse, it might reflect a trend to under-prophylact patients with a poor prognosis.

Whether there is an association between prophylaxis intensity and the occurrence of CIN/FN-related chemotherapy disturbances remains unclear, at least partially. Whether at the chemotherapy regimen level or the chemotherapy cycle level, under-prophylaxis was associated with an elevated risk of either dose reductions and delayed or canceled chemotherapy sessions.

The safety analyses revealed no differences in the rates of clinical events between the three prophylaxis intensity cohorts. The exception was headache, which was reported with greater frequency in correctly- and over-prophylacted patients. This might be related to the fact that underprophylacted patients received secondary prophylaxis and therefore had less drug exposure. Likewise, the rates of adverse drug reactions observed across cycles were similar across the three cohorts. The safety profile corresponds to what is known about standard filgrastim in general and biosimilar filgrastim in particular [18].

The analyses reported here warrant some caution in addition to limitations identified in our prior reports on the MONITOR-GCSF study [5, 6]. We classified patients into three levels of prophylaxis intensity, and further gradations might be possible. However, this may render comparative analyses more unwieldly if not overwhelming and yield differentiations that may not be clinically meaningful. Future analyses should also attempt to identify alternate methods for classifying patients into prophylaxis intensity categories that go beyond the amended EORTC assessment algorithm and take into account on-treatment data and markers. Our analyses were associative and the MONITOR-GCSF study was not designed to compare the effect of different levels of prophylaxis intensity to each other and an untreated control group. Such a comparison may not show a difference in outcome, and randomized controlled trials are necessary to evaluate the impact of differential prophylaxis on CIN/FN outcomes. We had the benefit of a large sample size. While lending statistical power to the study, it may also yield statistically significant results that may not necessarily be clinically meaningful.

The conclusion that clinicians' prophylaxis decisions may have been driven in part by patient-specific factors implies that, methodologically, there may have been a patient selection bias. However, by the same token and perhaps more relevant from a real-world evidence point of view, this may reflect routine clinical practice. This underscores the external validity of our findings.

\section{Conclusion}

The real-world evidence provided by the MONITORGCSF study indicates that providing GCSF support may yield better CIN, FN, and CIN/FN-related hospitalization outcomes if patients are prophylacted at levels above guideline recommendations. In contrast, patients who received inadequate GCSF prophylaxis are at markedly higher risk for poor outcomes. Our analyses show that guidelines may not be followed due to clinicians expectation of therapeutic benefit and clinical outcome, and provide real-world evidence to be integrated into future guidelines for GCSF prophylaxis in patients with solid tumors and hematological malignancies. 


\section{Compliance with ethical standards}

Disclosures C.B., P.G., M.A., H.L., and M.B. received compensation from Sandoz Biopharmaceuticals for their participation in the work reported here. M.G. was and A.K. is an employee of Hexal AG. K.D., I.A., and K.M. are affiliated with Matrix45. By company policy, they cannot hold equity in sponsor organizations and cannot receive direct personal benefits, financial or other, from sponsor organizations. Matrix45 provides similar services to other biopharmaceutical companies without exclusivity constraints.

Funding This work was supported by Hexal AG. Sponsor participated in the development of the protocol, implementation of the study, discussion of the results, and review of the manuscript for scientific content.

Open Access This article is distributed under the terms of the Creative Commons Attribution-NonCommercial 4.0 International License (http:// creativecommons.org/licenses/by-nc/4.0/), which permits any noncommercial use, distribution, and reproduction in any medium, provided you give appropriate credit to the original author(s) and the source, provide a link to the Creative Commons license, and indicate if changes were made.

\section{References}

1. Aapro MS, Bohlius J, Cameron DA et al (2011) 2010 update of EORTC guidelines for the use of granulocyte- colony stimulating factor to reduce the incidence of chemotherapy-induced febrile neutropenia in adult patients with lymphoproliferative disorders and solid tumours. Eur J Cancer 47:8-32

2. National Comprehensive Center Network (2015) 2015 NCCN Clinical Practice Guidelines in Oncology (NCCN Guidelines $\left.{ }^{\circledR}\right)$. Myeloid growth factors version 1.2015. www.ncen. org/professionals/physician_gls/pdf/myeloid_growth.pdf Accessed 8 Nov 2015

3. Gascón P, Aapro M, Ludwig H et al (2011) Background and methodology of MONITOR-GCSF, a pharmaco-epidemiological study of the multi-level determinants, predictors, and clinical outcomes of febrile neutropenia prophylaxis with biosimilar granulocyte-colony stimulating factor filgrastim. Critical Reviews in Oncology and Hematology 77:184-197

4. Gascón P, Aapro M, Ludwig H et al (2011) Update on the MONITOR-GCSF study of biosimilar filgrastim to reduce the incidence of chemotherapy-induced febrile neutropenia in cancer patients: protocol amendments. Critical Reviews in Oncology and Hematology 77:198-200

5. Gascón P, Aapro M, Ludwig H et al (2016) Treatment patterns and outcomes in the prophylaxis of chemotherapy-induced (febrile) neutropenia with biosimilar filgrastim (the MONITOR-GCSF study). Support Care Cancer 24:911-925 (Erratum published Support Care Cancer 2016;24:927)

6. Aapro M, Ludwig H, Bokemeyer C et al (2016) Predictive modeling of the outcomes of chemotherapy-induced (febrile) neutropenia prophylaxis with biosimilar filgrastim (MONITOR-GCSF study). Ann Oncol 27:2039-2045

7. Twisk JWR (2013) Applied longitudinal data analysis for epidemiology: a practical guide, 2nd edn. Cambridge University Press, Cambridge

8. Aapro M, Cameron D, Pettengell R et al (2006) EORTC guidelines for the use of granulocyte-colony stimulating factor to reduce the incidence of chemotherapy-induced febrile neutropenia in adult patients with lymphomas and solid tumours. Eur J Cancer 42:24332453

9. Crawford J, Dale D, Lyman GH (2003) Chemotherapy-induced neutropenia. Cancer 100:228-237

10. Kuderer N, Dale D, Crawford J et al (2007) Impact of primary prophylaxis with granulocyte colony-stimulating factor or febrile neutropenia and mortality in adult cancer patients receiving chemotherapy: a systematic review. J Clin Oncol 25:3158-3167

11. Lyman GH (2005) Guidelines of the National Comprehensive Cancer Network on the use of myeloid growth factors with cancer chemotherapy: a review of evidence. J Natl Compr Cancer Netw 3: 557-571

12. Gridelli C, Aapro M, Barni S et al (2007) Role of colony stimulating factors (CSFs) in solid tumours: results of an expert panel. Critical Reviews in Oncology and Hematology 63:53-64

13. Klastersky J, Awada A, Aoun M, Paesmans M (2009) Should the indications for the use of myeloid growth factors for the prevention of febrile neutropenia in cancer patients be extended? Curr Opin Oncol 21:297-302

14. Klastersky J, Awada A (2011) Prevention of febrile neutropenia in chemotherapy-treated cancer patients: Pegylated versus standard myeloid colony stimulating factors. Do we have a choice? Critical Reviews in Oncology and Hematology 78:17-23

15. Lyman GH, Dale DC, Culakova E et al (2013) The impact of the granulocyte colony-stimulating factor on chemotherapy dose intensity and cancer survival: a systematic review and meta-analysis of randomized controlled trials. Ann Oncol 24:2475-2484

16. Lyman GH, Abella E, Pettengell R (2014) Risk factors for febrile neutropenia among patients with cancer receiving chemotherapy: a systematic review. Critical Reviews in Oncology and Hematology 90:190-199

17. Weycker D, Hackett J, Edelsberg JS et al (2006) Are shorter courses of filgrastim prophylaxis associated with increased risk of hospitalization? Ann Pharmacother 40:402-407

18. Abraham I, Tharmarajah S, MacDonald K (2013) Clinical safety of biosimilar recombinant human granulocyte colony stimulating factors. Expert Opin Drug Saf 12:235-246 